\title{
Consumo de Camellia sinensis em população de origem oriental e incidência de doenças crônicas
}

\section{Camellia sinensis consumption by an Asian-Brazilian population and incidence of chronic diseases}

\author{
Fernanda FARIA \\ Rosane de Souza SANTOS ${ }^{1}$ \\ Lucia Marques VIANNA ${ }^{1}$
}

\section{RE S U M O}

Investigar os prováveis benefícios dos hábitos culturais orientais, incluindo o consumo de Camellia sinensis (chá verde) na prevenção de doenças crônicas. Trinta e quatro indivíduos entre 25 e 84 anos, de ambos os sexos, pertencentes à comunidade nipônica do Rio de Janeiro, RJ, foram submetidos a anamnese alimentar e questionário socio-econômico-cultural. Dos indivíduos, $76 \%$ consumiam chá verde, sendo que $35 \%$ o ingeriam diariamente e $27 \%$ esporadicamente. Verificou-se que o consumo regular de álcool era predominante nos não usuários de chá verde, quando comparados com os apreciadores da bebida (50\% e 35\%, respectivamente, faziam uso de álcool). Não foi observado sedentarismo na amostra. Dos apreciadores do chá, $81 \%$ não apresentavam doenças crônicas. Já entre os consumidores, observamos um menor percentual de doenças crônicas, especialmente, de hipertensão, porém sem significância estatística.

Termos de indexação: chá verde; doença crônica; epidemiologia; hábitos alimentares.

\section{A B S T R A C T}

To investigate the probable benefits of Asian dietary habits, including the intake of Camellia sinensis (green tea), in preventing chronic-degenerative diseases. Thirty-four individuals aging from 25 to 84 years from a Japanese community in Rio de Janeiro answered a socioeconomic-cultural questionnaire. Seventy-six percent of the individuals consumed green tea, where $35 \%$ consumed it daily and $27 \%$ consumed it sporadically. The regular intake of alcohol predominated among the non-consumers of green tea, when compared with green tea consumers (50\% and 35\%, respectively consumed alcoholic beverages). None of the individuals in the sample had a sedentary lifestyle. Among the individuals who consumed green tea, 81\% did not have any chronic illnesses. The consumers of tea presented a lower incidence of chronic diseases, especially hypertension, but it was not statistically significant.

Indexing terms: green tea; chronic diseases; epidemiology; food habits.

\footnotetext{
1 Laboratório de Investigação em Nutrição e Doenças Crônico-Degenerativas, Universidade Federal do Estado do Rio de Janeiro. Rua Xavier Sigaud, 290, Térreo, Urca, 22290-180, Rio de Janeiro, Brasil. Correspondência para/Correspondence to: L.M. VIANNA. E-mail: <lindcd@ig.com.br>.
} 


\section{N T R O D U Ç Ã O}

Os hábitos alimentares têm sido relacionados a fatores de risco para doenças crônicas'. Acredita-se, também, que a dieta exerça um papel fundamental na melhora da resposta imunológica, no retardo do envelhecimento e na prevenção de inúmeras doenças.

No Mediterrâneo e no Japão, por exemplo, postula-se que os padrões alimentares confiram benefícios à saúde. A dieta tradicional mediterrânea é baseada no consumo de óleo de oliva, peixes, grandes quantidades de frutas e vegetais, oleaginosas e cereais ${ }^{2}$, que contribuem para a baixa incidência de cânceres, relacionados a gorduras ${ }^{3}$, e de doenças coronarianas ${ }^{4}$, devido à presença de antioxidantes ${ }^{5}$ e à elevada proporção dietética de ácidos graxos monoinsaturados, em relação à de ácidos graxos saturados.

O padrão alimentar norte-americano, que tende a ser rico em gorduras e pobre em frutas e vegetais, exerce um papel negativo à saúde, acarretando um aumento dos riscos de doenças crônicas ${ }^{6}$.

A dieta japonesa se constitui no baixo consumo de gorduras e óleos em geral e, particularmente, na baixa taxa de ácido linoléico (n-6) em relação à de ácido linolênico (n-3), o que se atribui à larga ingestão de pescados. Destaca-se também o consumo de vegetais, frutas, soja e seus derivados, além do chá verde, que é consumido em grande proporção. Esse padrão dietético pode explicar a redução dos riscos de cânceres e doenças cardiovasculares, bem como, o fato da expectativa de vida nessa região ser a maior do mundo $3,7,8$.

Em relação à Camellia sinensis, várias propriedades fisiológicas vêm sendo atribuídas, porém encontramos uma certa inconsistência na literatura, traduzida por resultados controversos em relação ao uso do chá verde, quanto aos seus reais benefícios in vivo na redução da pressão $\operatorname{arteria|}^{9,10}$.

Assim, este trabalho teve como objetivo investigar os prováveis benefícios dos hábitos culturais orientais, incluindo o consumo de
Camellia sinensis (chá verde) na prevenção das doenças crônicas.

\section{MÉ T O D OS}

Foi realizado um estudo observacional analítico, no município do Rio de Janeiro, RJ, no período de janeiro a abril de 2003. O protocolo de pesquisa correspondente foi previamente aprovado pelo Comitê de Ética para Pesquisas em Humanos, do Departamento de Nutrição Aplicada da Universidade Federal do Estado do Rio de Janeiro.

A população de estudo foi composta por japoneses nativos ou descendentes de japoneses, residentes na cidade.

Para a execução da pesquisa, realizou-se junto a associações culturais, desportivas e religiosas japonesas, um levantamento preliminar dos indivíduos de ascendência oriental, vinculados a tais instituições.

Foram entrevistados 36 indivíduos que aceitaram responder o questionário, de ambos os sexos, com idades que variavam entre 25 e 84 anos, pertencentes à comunidade nipônica.

Foram aplicados questionários com variáveis socioeconômicas, clínicas, alimentares e culturais, relativas ao uso e freqüência de consumo de chá verde, modo de preparo da bebida, nível de prática de atividade física, nacionalidade ou ao grau de ascendência japonesa, uso de bebidas alcoólicas, tabagismo e histórico de doenças crônicas.

Os dados foram descritos por meio de proporções e analisados, quanto à significância estatística pelo teste Qui-Quadrado. O nível de significância estabelecido foi o valor de $p<0,05$.

\section{RES U L T A D O S}

Dos 36 entrevistados, a média de idade era de 54,4 $\pm 11,64$ anos (homens: 52,08 $\pm 11,26$ anos e mulheres: $55,63 \pm 11,9$ anos e $p=0,4035$ ). De fato, 34 foram incluídos na análise, tendo sido excluídos dois indivíduos que não preencheram apropriadamente os dados relativos ao sexo e à idade. 
Na amostra, nota-se um alto consumo de chá verde $(76 \%)$ entre os entrevistados. Em relação à freqüência com que o chá de Camellia sinensis era utilizado, verificou-se consumo diário da bebida por 35\% dos indivíduos, e consumo raro por $27 \%$ dos indivíduos.

Em relação aos hábitos culturais nipônicos, a Tabela 1 poderia indicar que o consumo regular de álcool foi superior no grupo de não apreciadores de chá verde (50\%), comparados aos usuários da erva (35\%), porém sem significância estatística.

Não foi observado sedentarismo, tanto na população de consumidores do chá quanto no grupo não apreciador da infusão, 65\% e 87\%, respectivamente, praticavam exercícios.

Nota-se que o aparecimento de doenças crônicas não se correlacionava à prática de exercícios físicos, uma vez que $71 \%$ dos indivíduos afirmavam praticar pelo menos algum tipo de atividade física e $80 \%$ dos indivíduos, que afirmavam ser sedentários, manifestavam algum tipo de doença crônica.

Na Tabela 2 observa-se a relação entre o consumo de chá verde e a presença de doenças crônicas, em que $81,0 \%$ dos indivíduos que relatavam consumir o chá não apresentavam quaisquer dessas doenças. Contudo, 50,0\% dos entrevistados que não faziam uso do chá verde registravam algum tipo de enfermidade crônica. Apesar disto, tais dados também não apresentaram significância estatística.

Tabela 1. Distribuição da população nipônica $(n=34)$, segundo a comparação entre os hábitos culturais do consumo de Camellia sinensis. Rio de Janeiro, 2003.

\begin{tabular}{|c|c|c|c|c|c|c|c|c|}
\hline \multirow{3}{*}{ Aspectos culturais } & \multicolumn{4}{|c|}{ Consome chá } & \multicolumn{4}{|c|}{ Não Consome chá } \\
\hline & \multicolumn{2}{|c|}{ Sim } & \multicolumn{2}{|c|}{ Não } & \multicolumn{2}{|c|}{ Sim } & \multicolumn{2}{|c|}{ Não } \\
\hline & $\mathrm{n}$ & $\%$ & $\mathrm{n}$ & $\%$ & $\mathrm{n}$ & $\%$ & $\mathrm{n}$ & $\%$ \\
\hline Consumo de álcool* & 9 & 35 & 17 & 65 & 4 & 50 & 4 & 50 \\
\hline Sedentarismo** & 9 & 35 & 17 & 65 & 1 & 13 & 7 & 87 \\
\hline Tabagismo*** & 0 & 0 & 26 & 100 & 1 & 13 & 7 & 87 \\
\hline
\end{tabular}

*Foram considerados consumidores de álcool aqueles que faziam uso de qualquer tipo de bebida alcoólica, regularmente. $\chi^{2}=0,610(p>0,25)$. ** Foram considerados sedentários os indivíduos que não praticavam exercícios físicos, regularmente. $\chi^{2}=1,748(0,25<p<0,1) ; * * * \chi^{2}=3,429$ $(0,1<p<0,05)$.
Tabela 2. Distribuição da população nipônica $(n=34)$, segundo associação entre doenças crônicas e consumo de chá verde. Rio de Janeiro, 2003.

\begin{tabular}{|c|c|c|c|c|c|c|}
\hline \multirow{3}{*}{ Doenças crônicas } & \multicolumn{4}{|c|}{ Chá } & \multirow{2}{*}{\multicolumn{2}{|c|}{ Total }} \\
\hline & \multicolumn{2}{|c|}{ Consome } & \multicolumn{2}{|c|}{ Não consome } & & \\
\hline & $n$ & $\%$ & $n$ & $\%$ & $n$ & $\%$ \\
\hline \multicolumn{7}{|l|}{ Sim* } \\
\hline Homens & 2 & 67,0 & 1 & 33,0 & 3 & 33,0 \\
\hline Mulheres & 3 & 50,0 & 3 & 50,0 & 6 & 67,0 \\
\hline Subtotal & 5 & 19,0 & 4 & 50,0 & 9 & 26,0 \\
\hline \multicolumn{7}{|l|}{ Não** } \\
\hline Homens & 7 & 78,0 & 2 & 22,0 & 9 & 75,0 \\
\hline Mulheres & 14 & 87,5 & 2 & 12,5 & 16 & 73,0 \\
\hline Subtotal & 21 & 81,0 & 4 & 50,0 & 25 & 74,0 \\
\hline Total & 26 & 100,0 & 8 & 100,0 & 34 & 100,0 \\
\hline
\end{tabular}

${ }^{*} \chi^{2}=0,227(0,9<p<0,5) ;{ }^{* *} \chi^{2}=0,464(0,5<p<0,1)$.

Tabela 3. Distribuição da população nipônica $(n=34)$, segundo a correlação entre os tipos de doenças apresentadas e o consumo de chá verde. Rio de Janeiro, 2003.

\begin{tabular}{lcccccc}
\hline \multirow{2}{*}{ Doenças crônicas } & \multicolumn{2}{c}{ Consome chá } & & \multicolumn{2}{c}{ Não consome chá } \\
\cline { 2 - 3 } \cline { 6 - 7 } & $\mathrm{n}$ & $\%$ & & $\mathrm{n}$ & $\%$ \\
\hline Hipertensão & 3 & 43 & & 4 & 57 \\
Diabetes Mellitus & 1 & 100 & & 0 & 0 \\
Outras & 1 & 100 & & 0 & 0 \\
\hline
\end{tabular}

${ }^{*} \chi^{2}=0,073(0,25<p<0,1)$.

A hipertensão arterial foi a principal doença mencionada entre os indivíduos, que não tinham como hábito o consumo de chá verde, atingindo $57 \%$ dos casos (Tabela 3).

\section{I S CUSS Ã O}

Ao avaliar a população estudada, percebe-se que o elevado consumo do chá de Camellia sinensis (chá verde), tanto por japoneses nativos quanto por descendentes desses, mesmo fora de seu país de origem, está inserido na cultura nipônica como um hábito tradicional. Os reflexos dessa cultura são observados, também, quando é analisada a ingestão de álcool, que foi bem menor entre os consumidores habituais do chá.

Achados científicos relatam que o consumo de álcool, o índice de massa corporal (IMC) e o 
fumo são determinantes importantes da pressão sangüínea em indivíduos japoneses ${ }^{11}$, sugerindo que o estilo de vida dessa população, em especial, mostra-se essencial para o desenvolvimento de doenças cardíacas.

Na amostra estudada, o sedentarismo não foi significante independentemente do consumo de chá. Curiosamente, a prática de atividade física pareceu não influenciar na incidência de doenças crônicas desse grupo. Entretanto, outros trabalhos, também não encontraram associação entre a prática de exercícios físicos e alterações das pressões arteriais sistólica e diastólica ${ }^{11}$. Adicionalmente, em recente estudo, igualmente, os autores não observaram ação protetora da prática do exercício físico sobre o perfil lipídico de japoneses de meia-idade e idosos ${ }^{12}$. Por outro lado, a literatura científica vem, constantemente, fortalecendo a ação benéfica dessas atividades na prevenção das doenças crônicas ${ }^{13,14}$.

Na realidade, efeitos fisiológicos benéficos à saúde humana vêm sendo atribuídos às catequinas presentes no chá verde, principalmente a epigalatocatequina-galato (EGCG), cuja distribuição é a mais abundante entre todos os componentes fenólicos existentes na planta.

Contudo, dados epidemiológicos sugerem que a associação entre o consumo regular de chá verde e a redução do risco do aparecimento de doenças cardiovasculares é conflitante e equivocada. Os efeitos fisiológicos do chá ou das catequinas do chá no estresse oxidativo, parecem ser proeminentes em modelos animais, porém acredita-se que a absorção das catequinas in vivo atinja baixas concentrações plasmáticas ${ }^{15,10}$. Ao mesmo tempo, a peroxidação lipídica in vivo, avaliada por meio da excreção urinária de $F(2)$ isoprostane, entre os consumidores de chá verde ( $273 \pm 48 \mathrm{pmol} / \mathrm{mmol}$ creatinina) e o grupo controle, que ingeria água quente $(263 \pm 47 \mathrm{pmol} / \mathrm{mmol})$, sugeriu que o consumo de chá verde não inibia a peroxidação lipídica ${ }^{16}$.

Por outro lado, existem evidências ${ }^{17}$ de que a oxidação de LDL - colesterol, relacionada ao risco de arteriosclerose e doenças cardíacas, seja inibida pelas catequinas presentes nesse chá.
Em um estudo realizado com 13916 indivíduos japoneses, com idades entre 40 e 69 anos, o consumo de chá verde foi associado com a redução dos níveis séricos de colesterol, quando esse consumo perfazia mais de 10 xícaras por dia, porém quando se relacionava com os aspectos de estilo de vida da população (tabagismo, uso de café e álcool), IMC e idade, a redução do colesterol foi observada com a ingestão de apenas uma xícara de chá por dia ${ }^{18}$.

Dados científicos já confirmaram que a hipertensão arterial é um dos maiores fatores de risco para muitas doenças cerebro-cardiovasculares, sendo também considerada uma das causas mais freqüentes de mortes no Japão ${ }^{19} \mathrm{e}$, que a ingestão regular de chá seja capaz de diminuir a pressão arterial. No entanto, o mecanismo pelo qual são exercidas as propriedades fisiológicas benéficas da bebida ainda permanece obscuro, como relatado em um estudo no qual observou-se uma relação inversa entre a pressão arterial sistólica e o consumo de chá, em que houve redução dos níveis pressóricos um $2,1 \mathrm{mmHg}$ e $3,5 \mathrm{mmHg}$, respectivamente em homens e mulheres saudáveis, sugerindo uma taxa de mortalidade associada à doença cardíaca coronariana maior, porém estatisticamente insignificante, entre os não consumidores da bebida e naqueles que consumiam menos de uma xícara de chá por dia ${ }^{20}$.

Um outro estudo ${ }^{21}$ investigou a relação da ingestão de chá e de um biomarcador de exposição aos polifenóis, derivados do chá verde, com a pressão sangüínea de 218 mulheres com mais de 70 anos, em que se observou uma associação entre a maior ingestão de chá, em conjunto com uma maior excreção urinária do referido biomarcador e níveis sistólicos significativamente menores.

\section{O N CLUS Ã O}

Esse trabalho, de acordo com o tamanho da amostra, não é suficiente para extrair conclusões definitivas sobre os benefícios dos hábitos orientais na prevenção de doenças crônicas na população nipônicas. 
Embora nossos resultados tenham revelado menor ocorrência de doenças crônicas no grupo de consumidores do chá, não houve significância estatística. Tais evidências, ainda que preliminares, estimulam a realização de estudos longitudinais para averiguar a efetividade do chá de Camellia sinensis na prevenção de doenças cardiovasculares.

\section{A G R A DE CIMENTO}

Ao apoio financeiro do Conselho Nacional de Desenvolvimento Científico e Tecnológico (CNPq).

\section{RE FERÊ NCIAS}

1. Kromhout D. Diet and cardiovascular diseases. J Nutr Health Aging. 2001; 5(3):144-9.

2. Wasling $C$. Role of the cardioprotective diet in preventing coronary heart disease. Br J Nurs. 1999; 8(8):1239-48.

3. Tokudome S, Nagaya T, Okuyama H, Tokodome N, Imaeda N, Kitagawa I, et al. Japanese versus Mediterranean diets and cancer. Asian Pacific J Cancer Prev. 2000; 1:61-6.

4. Trichopoulou A. Mediterranean diet: the past and the present. Nutr Metab Cardiovasc Dis. 2001; 11(4):1-4.

5. Barbaste M. Dietary antioxidants, peroxidation and cardiovascular risks. J Nutr Health Aging. 2002; 6(3):209-23.

6. Satia-Abouta J. Dietary acculturantion: applications to nutrition research and dietetics. J Am Diet Assoc. 2002; 102(8):1105-18.

7. Sugano M. Characteristics of fats in Japanese diets and current recommendations. Lipids. 1996; 31 Suppl:S283-6.

8. Hinohara S. On the lifestyle of the aged from the view point of holistic medicine. Seishin Shinkeigaku Zasshi. 2002; 104(12):1160-6.

9. Vinson J. Black and green tea and heart disease: a review. Biofactors. 2000; 13(1-4):127-32.

10. Riemersma R, Rice-Evans C, Tyrrell R, Clifford M, Lean M. Tea flavanoids and cardiovascular health. QJM. 2001; 94(5):277-82.

11. Kono S, Schinchi K, Ikeda N, Yanai F, Imanishi K, Handa K. Lifestyles, glucose tolerance and blood pressure in male self-defence officials in Northen Kyshu, Japan. J Hum Hypertens. 1992; 6(2):101-5.

12. Fujino Y, Mizoue T, Tokui N, Yoshimura T. Walking exercise and its relationship to serum lipids in Japanese. J Epidemiol. 2002; 12(2):64-9.

13. Nordstrom C, Dwyer K, Merz C, Shircore A, Dwyer J. Leisure time physical activity and early atherosclerosis: the Los Angeles Atherosclerosis Study. Am J Med. 2003; 115(1):19-25.

14. Thompson $P$, Buchner D, Pina I, Balady G, Williams $M$, Marcus B. Exercise and physical activity in the prevention and treatment of atherosclerotic cardiovascular disease: a statement from the Council on Clinical Cardiology (Subcommittee on Exercise, Rehabilitation, and Prevention) and the Council on Nutrition, Physical Activity, and Metabolism (Subcommittee on Physical Activity). Circulation. 2003; 107(24):3109-16.

15. Higdon J, Frei B. Tea catechins and polyphenols: health effects, metabolism, and antioxidant functions. Crit Ver Food Sci Nutr. 2003; 43(1): 89-143.

16. Hodgson J, Croft K, Mori T, Burhe V, Beilin L, Puddey I. Regular ingestion of tea does not inhibit in vivo lipid peroxidation in humans. J Nutr. 2002; 132(1):55-8.

17. Weisburger J, Chung F. Mechanisms of chronic disease causation by nutritional factors and tobacco products and their prevention by tea polyphenols. Food Chem Toxicol. 2002; 40(8):1145-54.

18. Tokunaga S, White I, Frost C, Tanaka K, Tokudome S, Akamatsu T, et al. Green tea consumption and serium lipids and lipoproteins in a population of healthy workers in Japan. Ann Epidemiol. 2002; 12(3):157-65.

19. Yasujima M. Guidelines for the managnetment of hypertension in Japan. Rinsho Byori. 2003; 51(5):581-5.

20. Stensvold I, Tverdal A, Solvoll K, Foss O. Tea consumption, relationship to cholesterol, blood pressure, and coronary and total mortality. Prev Med. 1992; 21(4):546-53.

21. Hodgson J, Devine A, Puddey I, Chan S, Beilin L, Prince $\mathrm{R}$. Tea intake is inversely related to blood pressure in older women. J Nutr. 2003; 133(9):2883-6.

Recebido em: 21/5/2004

Versão final reapresentada em: 21/3/2005

Aprovado em: 29/6/2005 